\title{
Design and Manufacturing of a Body-Powered Hook with Force Regulation System and Composite-Based Nanomaterials
}

\author{
Manuel Alejandro Trejo-Letechipia ${ }^{1, *(\mathbb{D})}$, David Arturo Rodriguez-Sanchez ${ }^{1}$, Reyna Berenice González-González ${ }^{1}$, \\ Alberto Isaac Perez-Sanpablo ${ }^{2}$, , Ana María Arizmendi-Morquecho ${ }^{3}$, Tania E. Lara-Ceniceros ${ }^{3}$ (1), \\ Jose Bonilla-Cruz $^{3}{ }^{-1}$ and Jorge Armando Cortes-Ramirez ${ }^{1}$ \\ 1 Tecnologico de Monterrey, Escuela de Ingeniería y Ciencias, Monterrey 64849, Mexico; \\ A01056340@itesm.mx (D.A.R.-S.); A00818415@itesm.mx (R.B.G.-G.); jcortes@tec.mx (J.A.C.-R.) \\ 2 National Institute of Rehabilitation Luis Guillermo Ibarra Ibarra, Ciudad de México 14389, Mexico; \\ albperez@inr.gob.mx \\ 3 Advanced Functional Materials \& Nanotechnology Group, Centro de Investigacioń en Materiales Avanzados \\ S. C. (CIMAV-Unidad Monterrey), Apodaca 66628, Mexico; ana.arizmendi@cimav.edu.mx (A.M.A.-M.); \\ tania.lara@cimav.edu.mx (T.E.L.-C.); jose.bonilla@cimav.edu.mx (J.B.-C.) \\ * Correspondence: a01061265@itesm.mx
}

Citation: Trejo-Letechipia, M.A.;

Rodriguez-Sanchez, D.A.;

González-González, R.B.;

Perez-Sanpablo, A.I.;

Arizmendi-Morquecho, A.M.;

Lara-Ceniceros, T.E.; Bonilla-Cruz, J.;

Cortes-Ramirez, J.A. Design and

Manufacturing of a Body-Powered

Hook with Force Regulation System and Composite-Based Nanomaterials. Appl. Sci. 2021, 11, 4225. https:// doi.org/10.3390/app11094225

Academic Editor: José

A. González-López

Received: 11 April 2021

Accepted: 30 April 2021

Published: 6 May 2021

Publisher's Note: MDPI stays neutral with regard to jurisdictional claims in published maps and institutional affiliations.

Copyright: (c) 2021 by the authors. Licensee MDPI, Basel, Switzerland. This article is an open access article distributed under the terms and conditions of the Creative Commons Attribution (CC BY) license (https:// creativecommons.org/licenses/by/ $4.0 /)$.
Abstract: New developments in upper limb prostheses are based almost exclusively on myoelectric technology, which prioritizes the needs and infrastructure of developed countries. However, $80 \%$ of people with disabilities living in developing countries cannot afford these devices. The main needs of users in developing countries were identified through the I-Corps program, to select the ideal prosthesis type and generate a list of design requirements to improve prosthetic performance. A body-powered hook was developed through a four-stage process: conceptual design, detailed design, design for manufacturing, and manufacturing. The result was the lightweight body-powered hook with a voluntary open mechanism; it weighed $100 \mathrm{~g}$ and was manufactured on carbon fiber enriched with nanomaterials to provide hydrophobic and antibacterial properties. Furthermore, this prosthesis contains a novel continuous force regulation system, which allows a constant griping force from 0 to $30 \mathrm{~N}$, providing the user with proprioceptive force to enable accurate and quick-responsive control. Due to its high functionality, robustness and comfort, the upper limb prosthesis developed in this study is an appropriate alternative for most users, regardless of their economic condition or the infrastructure of their country.

Keywords: prosthetics; prosthetic hand; body-powered

\section{Introduction}

Humans have relied heavily on the use of their hands throughout history, since they provide a wide range of problem-solving possibilities. Therefore, replacing an upper limb represents an enormous technological challenge. Despite the large number of available prosthetic alternatives, such as a myoelectric hand, body-powered hand, passive hand, and body-powered hook, among others, prosthesis rejection rate (41-74\%) remains high [1].

It is worth noting that $80 \%$ of the global population of more than 600 million people with disabilities live in developing countries, demonstrating a significantly higher prevalence of disability than in their high-income counterparts [2,3]. However, current research on the development of medical devices prioritizes the needs and infrastructure of developed countries [2]. Furthermore, high-tech myoelectric prostheses have recently been presented as the most attractive alternative due to their stylized appearance and broad variety of functions [4]. Nonetheless, the majority of consumers cannot afford such technology. Consequently, an imbalance has emerged between the wide diversity of high-tech devices for developed countries and the relative lack of appropriate prosthetics for developing countries [2]. Given these circumstances, it is imperative for new prosthetic research to focus on the needs of the vast majority of users. 
Body-powered technology is an appropriate alternative for those in developing countries, since it meets the crucial " $4 \mathrm{~A}^{\prime} \mathrm{s}$ " (availability, accessibility, appropriateness, and affordability) [5]. Interestingly, body-powered prostheses are popular in developed countries as well, where they are frequently preferred due to their high functionality, robustness, performance, and light weight [1,6-8]. A higher functionality was demonstrated in $\mathrm{Cy}$ bathlon [9], a competition that evaluates the functionality of medical devices. For the upper-limb prostheses category, recent experimental designs competed against the most advanced prostheses available on the market. Although prostheses such as Michelangelo [10], BeBionic [10], and I-Limb [11] were competing, the prosthesis with the highest valuation in Cybathlon 2016 and Cybathlon 2020 was a body-powered prosthesis [12], which shows that a simpler technology does not necessarily mean less functionality. Thus, the evolution of body-powered technology will have an impact not only on developing countries but also on developed ones.

Hands or hooks are used as terminal devices on body-powered prostheses. Although body-powered hands have a better appearance, their use for daily-living activities is limited; body-powered hooks, in contrast, are ideal for day-to-day functions, since they have higher functionality, lower weight [13], direct feedback, robustness, and low acquisition and maintenance costs [14], which make them preferred by users $[6,15]$. The technology of a body-powered hook is simple: there are no sensors, batteries, or electrical/electronic components. Therefore, the user controls the opening and closing of the prosthesis through body movement (typically by shoulder abduction). There are two categories of bodypowered devices: voluntarily closed (VC) and voluntarily open (VO). VC devices are normally open, and user activation closes them. Once released, an elastic band restores the system to its open state [16]. Their main advantage is that users can control the grip force; however, they must maintain the grip force to hold an object, causing fatigue and discomfort [13]. In contrast, $\mathrm{VO}$ devices are normally closed, and user activation opens them. An elastic band closes the mechanism to its inactive position, which leads to more comfort, since the user only needs to apply force when they desire to open the prosthesis [17]. Grasping force is determined by the strength of the passive band; therefore, the user cannot hold objects that require more grip force than the band provides. In addition, as users select the passive band according to the maximum force they need, users expend an unnecessary amount of energy when they desire to hold a lightweight object that requires a lower pinch force [18]. To overcome these disadvantages, VO devices have been designed to allow adjustments, but only by limited number of discrete levels of grip force [18].

Body-powered hook technology has not evolved significantly over the last years [14,19]. A research study conducted more than two decades ago [20], reported the design priorities for body-powered technology users, and these user priorities are still present in surveys from more recent studies [1]. This lack of research and development leads to high rejection rates $[6,21]$. Despite the obvious need for new body-powered alternatives, only a few new developments have emerged recently, such as the "Maker hand" [22] and the "Delft cylinder hand" [23]. Both use the hand as a terminal device, which is not frequently used and has the highest rejection rate for body-powered technology [1]. In contrast, the BodyPowered Grip VC "GRIP 5" [24] has high functionality due to its VC mechanism, since the user can manipulate the pressure control. However, in daily use, the VC mechanism can cause fatigue and discomfort [13]. Contrarily to body-powered devices, there is a large variety of 3D-printed prostheses [25-27]. Three-dimensional printing facilitates the development process and allows a great variety of designs without the restrictions of typical manufacturing processes [28]. However, 3D-printed prostheses have not reported user acceptance, functionality, and durability [16]. Therefore, the context and needs of prosthetic users should be considered while designing prostheses [29].

In this study, we present the design and development of a prosthesis that meets the requirements and conditions for the majority of users. The designed "Carbon HK" is a body-powered hook VO, which incorporates ultra-light and resistant materials enriched 
with nanoparticles that provide antibacterial and hydrophobic properties. In addition, its installation is easy and presents a novel continuous force regulation system. Therefore, this study presents an appropriate medical device for the needs of the majority of users, which will reduce rejection rates and ensure an effective alternative to improve users' health.

\section{Materials and Methods}

\subsection{I-Corps Program and Design Process}

A lightweight and highly functional body-powered hook was designed as a result of the I-Corps program developed by the National Science Foundation (NSF) of the United States (Figure 1). The objective of the I-Corps methodology process is to provide developments tailored to the needs of a population. In this study, 106 interviews were conducted with users and related personnel (prosthetists, rehabilitators, sellers, administrative personnel of medical centers, and politicians) in order to identify the needs of the users from certain developing countries. In addition, how consumers typically use their prosthesis for daily activities was also analyzed. Based on the results of these analyses and a literature review, a list of requirements was defined to design a body-powered hook with a VO mechanism. The design process consists of four stages:

(1) Conceptual design: Various concepts proposals were created and evaluated. The most appropriate concept was selected according to the fulfillment of the previous established requirements.

(2) Detailed design: At this stage, the materials and the manufacturing processes of the parts were selected, as well as the purchase parts, the assembly sequence, and the kinematics of the mechanism. During this stage, possible problems in the selected concept were identified in order to make the pertinent changes to achieve an optimal design.

(3) Design for manufacturing: At this stage, the design was modified according to the manufacture process, as well as geometric dimensioning and tolerancing (GD\&T), which was used to achieve a quality that allows correct assembly. Then, the tools and fixtures required for manufacturing and assembly were constructed.

(4) Manufacturing: The manufacturing of the final design was performed.

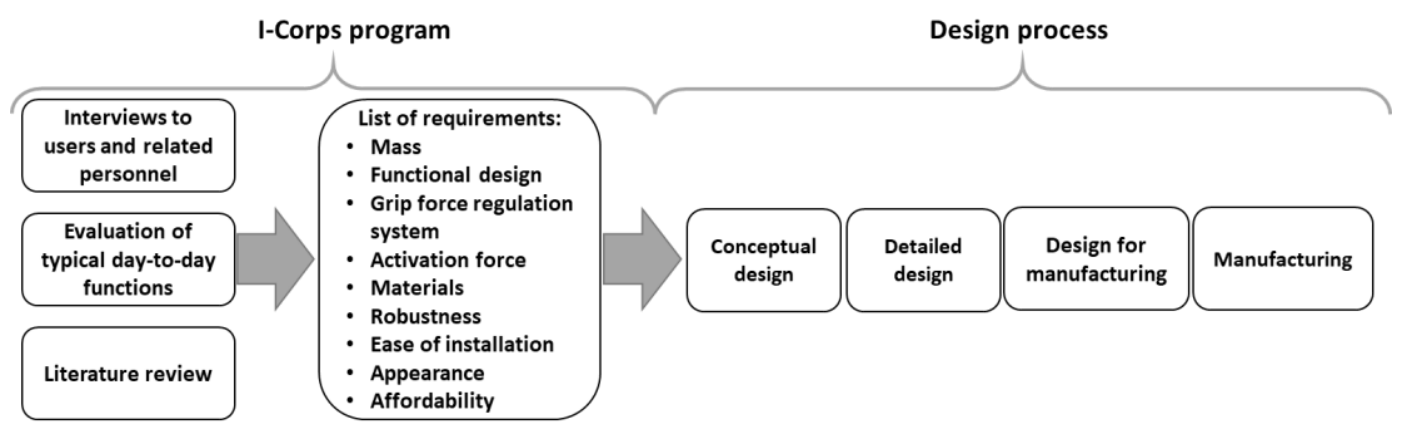

Figure 1. Methodology overview.

\subsection{List of Requirements}

The requirements to which the body-powered hook had to comply were identified according to the literature review, and are classified in the following criteria: mass, functional design, grip force regulation system, activation force, materials, robustness, ease of installation, appearance, and affordability. Each design criterion is described below.

\subsubsection{Mass}

The human hand has an average weight of $400 \pm 90 \mathrm{~g}$ [30]. Currently, various prostheses are below that weight; however, users still consider them too heavy because the perception of weight for the user is greater, since the prosthesis is not connected to the musculoskeletal system. Considering that the Hosmer 5X body-powered hook [31] (a 
commonly used prosthesis in developing countries) weighs $213 \mathrm{~g}$ and that the main desire of users is weight reduction [1], the weight of the prosthesis designed in this study was intended to be less than that of the Hosmer $5 \mathrm{X}$.

\subsubsection{Functional Design}

The human hand and wrist have 32 muscles and 15 links [32], which allow several movement patterns and, therefore, various functions. The most advanced myoelectric prostheses have independent movement in each finger; however, a higher functionality has not yet been proven in comparison to body-powered hook prostheses. Although this technology has not evolved in recent years [14], the body-powered hook provides the user with proprioceptive force and position feedback, enabling accurate and fast control. We propose a simple design for a basic hook grip and tip pinch grasp functions, which would allow the performance of the highest number of daily tasks.

\subsubsection{Grip Force Regulation System}

The human male hand can apply a gripping force of $80 \pm 20 \mathrm{~N}$ on a tip pinch position [32]. To enable daily-living activities, the prosthesis should have a gripper force above $20 \mathrm{~N}$ [14]. Commercial prostheses, such as the Hosmer 5XA, reported a maximum

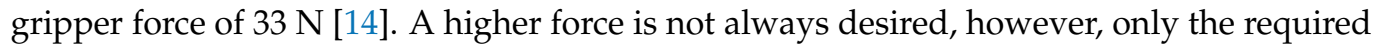
force to hold a specific object. In this manner, our objective is to design a prosthesis with a maximum gripper force of $30 \mathrm{~N}$, which has the ability to regulate the gripper force that allows the holding of delicate and heavy objects.

\subsubsection{Activation Force}

Body-powered prostheses require activation force through a cable operation, which is typically moved by shoulder abduction and can cause fatigue, discomfort, and pain for users. To avoid fatigue, the activation force should be below $38 \mathrm{~N}$ for the average female and $66 \mathrm{~N}$ for the average male [21]. A higher activation force is one of the reasons for prosthesis rejection [13]; however, commercial alternatives are typically above the desired level. For example, the activation force of the Ottobock Model 10A60 hook prosthesis is $101 \mathrm{~N}$, with a cable excursion of $35 \mathrm{~mm}$ [14]. Similarly, the activation force and the cable excursion of the Hosmer Model 5XA hook are $95 \mathrm{~N}$ and $46 \mathrm{~mm}$ [14], respectively. Thus, our objective is to design a prosthesis with a variable activation force that is selectable by the user, ranges from 0 to $66 \mathrm{~N}$ to avoid fatigue, and comes with a cable excursion of $45 \mathrm{~mm}$, since it is an adequate distance to be moved by shoulder abduction.

\subsubsection{Materials}

Commercial body-powered prostheses are generally made from stainless steel $(E \approx$ $\left.200 \mathrm{GPa}, \rho \approx 8 \mathrm{~g} / \mathrm{cm}^{3}\right)$ or aluminum $\left(E \approx 70 \mathrm{GPa}, \rho \approx 2.7 \mathrm{~g} / \mathrm{cm}^{3}\right)$. Carbon fiber $\left(E \approx 150-500 \mathrm{GPa}, \rho \approx 1.6 \mathrm{~g} / \mathrm{cm}^{3}\right)$ has become a popular material in various medical areas due to its low weight and high mechanical resistance, being $1 / 5$ lighter than steel and $1 / 2$ lighter than aluminum. Moreover, some new myoelectric upper limb prosthesis models use carbon fiber pieces. In this sense, carbon fiber is an ideal material for a new device. It should be noted that a hand prosthesis is in direct contact with various objects whose cleanliness cannot be ensured, as well as with food; as a result, it is essential that the material have antibacterial and/or repellent properties. Thus, the surface properties (i.e., hydrophobic and antibacterial) of the materials were analyzed. Contact angle measurements were performed with an optical contact angle apparatus (OCA 15 PLUS-Data Physics). Antibacterial property was tested against Gram-negative (E. coli) and Grampositive bacteria (S. aureus). Atomic force microscopy (AFM) images were obtained with a MFP3D-SA microscope (Asylum Research) in order to analyze the surface characteristics, such as roughness and nanoparticle dispersion. 


\subsubsection{Robustness}

A prosthesis must be designed to perform daily-life activities; therefore, its materials must withstand contact with water, earth, dust, sunlight, as well as other degrading agents. A robust device requires mechanical resistance to perform the tasks for which it was designed, and the consumable parts should be easily replaced.

\subsubsection{Ease of Installation}

The prosthesis must be designed to be easy to place in the stump socket, considering that it will be installed in workshops with limited equipment and low-qualified personnel.

\subsubsection{Appearance}

It is essential that the user feels satisfied with the appearance of the prosthesis; if the device is not to their liking, they will simply not use it. The metallic body-powered hooks have an unpleasant design which is cold to the touch; they also appear dangerous due to their sharp edges. The proposed design should not have sharp edges, should not be cold to the touch, and should have an attractive look.

\subsubsection{Affordability}

The design is intended to ensure the best possible quality at an affordable cost for the greatest number of people. The prosthesis should be suitable for markets in developed and developing countries and have a similar commercial value to prostheses of the same type.

\section{Results and Discussion}

\subsection{I-Corps Program}

Upper limb prosthesis are classified as follows: passive hook, passive hand, bodypowered hook, body-powered hand, electric hook, electric gripper, and electric hand, among others [1]. In order to design the appropriate prosthesis for the majority of users, this work began by selecting the type of prosthesis through the I-Corps program. This program consisted of open interviews in person and by telephone with 106 people from Mexico, Colombia, and Guatemala. Ten medical facilities were visited in the cities of Morelia, Monterrey, Guadalajara, Mexico City, and Puebla. In addition, three volunteers with direct experience concerning the challenges facing users on a day-to-day basis participated in the study and provided useful information. Some of the most significant comments from the interviews are presented in Table 1.

The results showed that, in Mexico, the use of electric prostheses is almost non-existent due to certain factors, such as the weather. The high temperatures and high humidity levels caused by the country's geographic location make it difficult for the user to wear a closed silicone socket. Furthermore, the connection between the socket and the myoelectric sensors is inadequate due to sweating. In Mexico, there is also no standardization of medical services. Most of the country has neither specialists nor the sufficient infrastructure necessary to place and maintain an electrical prosthesis. Moreover, the public health system in Mexico cannot afford this type of prosthesis. Thus, electric prostheses do not comply with any of the " 4 A's" (availability, accessibility, appropriateness, and affordability) in Mexico. Similarly, passive prostheses were not a good alternative either because the largest group of amputees in Mexico are male workers who require a highly functional prosthesis.

Regarding body-powered hand prostheses, the glove must be frequently replaced, and they have a limited functionality, even though they have a hand-shaped design. In contrast, body-powered hook prostheses are lighter, more functional, more robust, and more comfortable. Several users commented that they prefer body-powered hooks over body-powered hands. In addition, the National Rehabilitation Institute (INR, for its acronym in Spanish), which is the largest and most important rehabilitation center in Mexico, provides mainly body-powered hook prostheses. 
Table 1. Interviews with prosthetic users and related personnel.

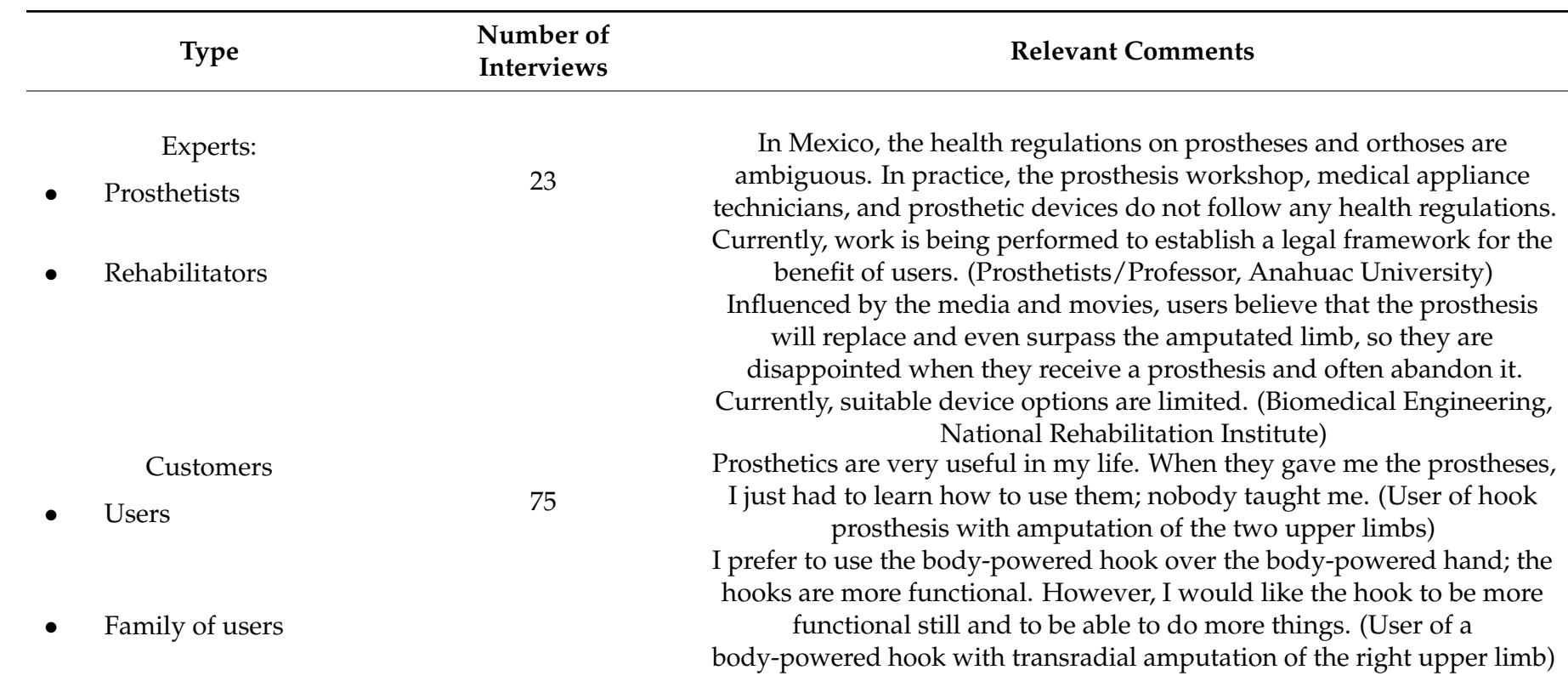

\section{Others}

- Sellers

- Administrative personnel of medical centers
There is no standardization in the quality of medical services in the national territory. In the capital of the country, there is infrastructure and trained personnel, but in the countryside, there is a lack of infrastructure and qualified personnel. So many patients do not receive the best service. (Bureaucrat, Mexican Institute of Social Security)

As a result of the I-Corps program, a body-powered hook was selected as the appropriate prosthesis. Some of its advantages are as follows: lower weight, higher functionality, lower cost, low maintenance, and user feedback, Moreover, a body-powered hook does not require highly equipped prosthetics workshops, nor does it require highly qualified personnel for its installation. Finally, the skin inside the stump socket can be covered with cotton, allowing perspiration. Furthermore, commercial prostheses of this type in Mexico comply with the " 4 A's".

\subsection{Design Process}

\subsubsection{Conceptual Design}

The Hosmer $5 \mathrm{X}$ by Fillauer was used as the study model, as it is the most widely used prosthesis in Mexico. The conceptual design of the gripper and hook with force regulation (Figure 2) was the result of the creation of various original concepts. The selected concept was designed with a hook shape that allows users to hold objects without activating the prosthesis. It has a mechanism to regulate the gripping force (Figure 3a) through the rotation of a worm gear, which tightens or releases a spring associated with the gripping force. It also has a laminar design, which facilitates its manufacture in carbon fiber. 


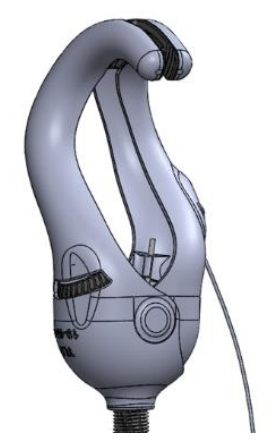

Figure 2. Conceptual design: gripper and hook with force regulation.

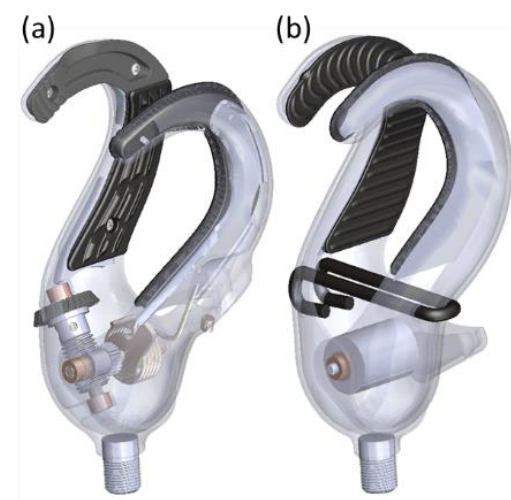

Figure 3. (a) Worm gear crown mechanism concept, (b) tubular band mechanism concept.

\subsubsection{Detailed Design}

Significant modifications were made to the selected conceptual design, mainly in the regulation of the force of the gripper, which was originally based on the rotation of a worm gear mechanism (Figure 3a). Since this concept had an exposed mechanism, it was vulnerable to dust damage, requiring a greater number of parts and a higher geometric tolerance. Therefore, a simpler "tubular band mechanism concept" was proposed by using a tubular band to regulate the gripper's force (Figure 3b). As a result, 16 parts were eliminated, including screws, gears, bushings, as well as the spring and shaft. The elimination of the gears increased the required geometric tolerances.

The mechanism and its dimensions were designed within the range of the measures presented by various commercial VO hand and hook prostheses [14]. Mechanically, the device has an opening of $50^{\circ} \theta$ (Figure 4), which allows a maximum clamp opening of $84 \mathrm{~mm}$ (from K to D point in Figure 4). In this way, the user is able to hold various objects. The maximum cable excursion of $42 \mathrm{~mm}$ shows that the device can be manipulated by the user through shoulder abduction. The design has the capacity to regulate grip force when adding tension to or releasing the tubular band.

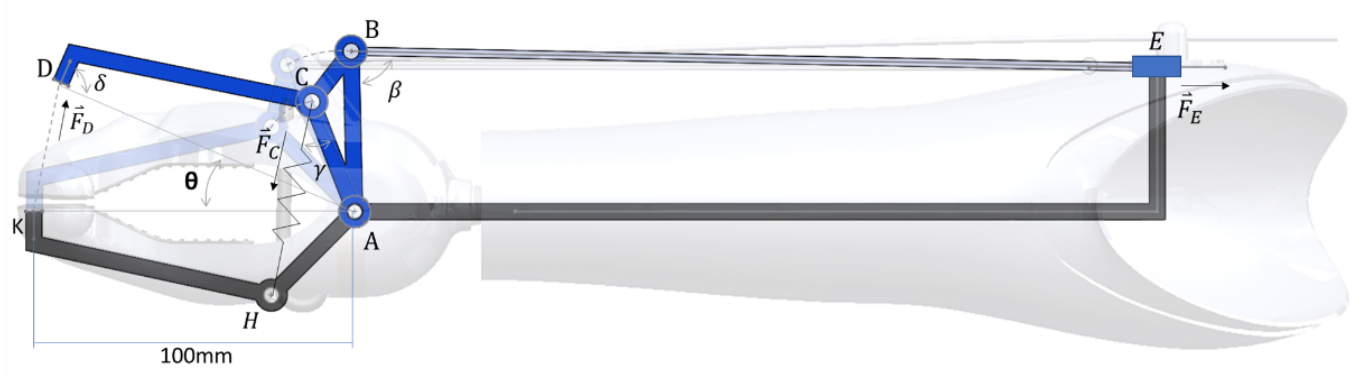

Figure 4. Kinematic diagram. 
A kinetic study was performed using a tubular band with a design specification $k$ of $5.0 \mathrm{~N} / \mathrm{mm}$ and a unstretched length of $31 \mathrm{~mm}, 43 \mathrm{~mm}$, and $47 \mathrm{~mm}$, attached from point $C$ to point $\mathrm{H}$ (Figure 4), which has an initial distance of $52 \mathrm{~mm}$ at $0^{\circ} \theta$. The activation and pinch force were determined by static equilibrium equations; that is, the principle that the sum of moments resulted by each force equals zero, as shown in Equation (1):

$$
\sum \vec{M}_{A}=\vec{M}_{c}-\vec{M}_{E}-\vec{M}_{D}=
$$

where $\sum \vec{M}_{A}$ stands for the moment that resulted in point A, while $\sum \vec{M}_{c}, \sum \vec{M}_{E}$, and $\sum \vec{M}_{D}$ stand for the moments generated by the pinch, the cable, and the elastic band, respectively. Then, each moment was decomposed into its components, as in: $\vec{M}_{i}=\vec{r}_{i} \times \vec{F}_{i}$. Where $\vec{r}_{i}$ represents the distance from the applied force to the point of rotation and $\vec{F}_{i}$ represents the actual force. Finally, each force was determined separately by considering one term equal to zero at a time. For $\vec{F}_{E}, \vec{M}_{D}=0$, when the cable is excursed, as shown in Equation (2):

$$
\sum \vec{M}_{A}=\vec{M}_{c}-\vec{M}_{E}=0 \rightarrow \vec{M}_{C}=\vec{M}_{E} \rightarrow \vec{r}_{C} \times \vec{F}_{c}=\vec{r}_{B} \times \vec{F}_{E} \rightarrow \vec{F}_{E}=\frac{\vec{r}_{C} \times \vec{F}_{C}}{\vec{r}_{B}}
$$

And $\vec{M}_{E}=0$ for $\vec{F}_{D}$, as seen in Equation (3):

$$
\sum \vec{M}_{A}=\vec{M}_{C}-\vec{M}_{D}=0 \rightarrow \vec{M}_{C}=\vec{M}_{D} \rightarrow \vec{r}_{C} \times \vec{F}_{C}=\vec{r}_{D} \times \vec{F}_{D} \rightarrow \vec{F}_{D}=\frac{\vec{r}_{C} \times \vec{F}_{C}}{\vec{r}_{D}}
$$

Now, $\vec{r}_{C}$ stands for the distance from points $\mathrm{A}$ to $\mathrm{C}$; it is equal to $36.77 \mathrm{~mm}$. $\vec{r}_{B}$ goes from $\mathrm{A}$ to $\mathrm{B}$ and equals $50 \mathrm{~mm}$. Finally, $\vec{r}_{D}$ goes from $\mathrm{A}$ to $\mathrm{D}$ and equals $100 \mathrm{~mm}$. $\vec{F}_{c}$ was defined by Hooke's law, $\vec{F}=k \vec{x}$, where $k$ is the elastic constant of the band and $\vec{x}$, its displacement. For simplicity's sake, $\vec{F}_{E}, \vec{F}_{D}$, and $\vec{F}_{C}$ were reduced to their perpendicular components, as shown in Equation (4):

$$
\sin (\beta) \vec{F}_{E}=\frac{\vec{r}_{C} \sin (\gamma) \vec{F}_{C}}{\vec{r}_{B}} \rightarrow \vec{F}_{E}=\frac{\vec{r}_{C} \sin (\gamma) \vec{F}_{C}}{\sin (\beta) \vec{r}_{B}}, \quad \sin (\delta) \vec{F}_{D}=\frac{\vec{r}_{C} \sin (\gamma) \vec{F}_{C}}{\vec{r}_{D}} \rightarrow \vec{F}_{D}=\frac{\vec{r}_{C} \sin (\gamma) \vec{F}_{C}}{\sin (\delta) \vec{r}_{D}}
$$

where $\gamma$ and $\beta$ are the angles between the elastic band and $\vec{r}_{C}$ and between the cable and $\vec{r}_{B}$, respectively. Regarding $\delta$, since $\vec{F}_{D}$ was assumed to be always perpendicular to $\vec{r}_{D}$, $\delta=90^{\circ} \therefore \sin \left(90^{\circ}\right)=1$.

The kinetic study results were validated through simulation. The values are plotted in Figure 5. The activation force presented in Figure 5a shows the magnitude of force $\vec{F}_{E}$ when $\vec{M}_{D}=0$. Similarly, the gripper force curve presented in Figure $5 \mathrm{~b}$ shows the magnitude of $\vec{F}_{D}$ when $\vec{M}_{E}=0$.

For the manufactured parts, carbon fiber was selected with a thickness of $1.8 \mathrm{~mm}$, which was manufactured by autoclave molding. Liquid silicone rubber manufactured by molding cast was used for the clamping rubbers. Yet, for the purchase parts, the following materials were selected: sintered bronze bearings with an inner diameter tolerance of $+0.012 / 0$; a stainless steel AISI 416 shaft with a tolerance of $-0.004 /-0.012$; a stainless steel screw of $1 / 2$ "; and a rubber tubular band with a $\mathrm{K}$ of $5.0 \mathrm{~N} / \mathrm{mm}$ (Figure $3 \mathrm{~b}$ ). Finally, the feasibility of the assembly sequence was successfully validated by a virtual assembly sequence test. 


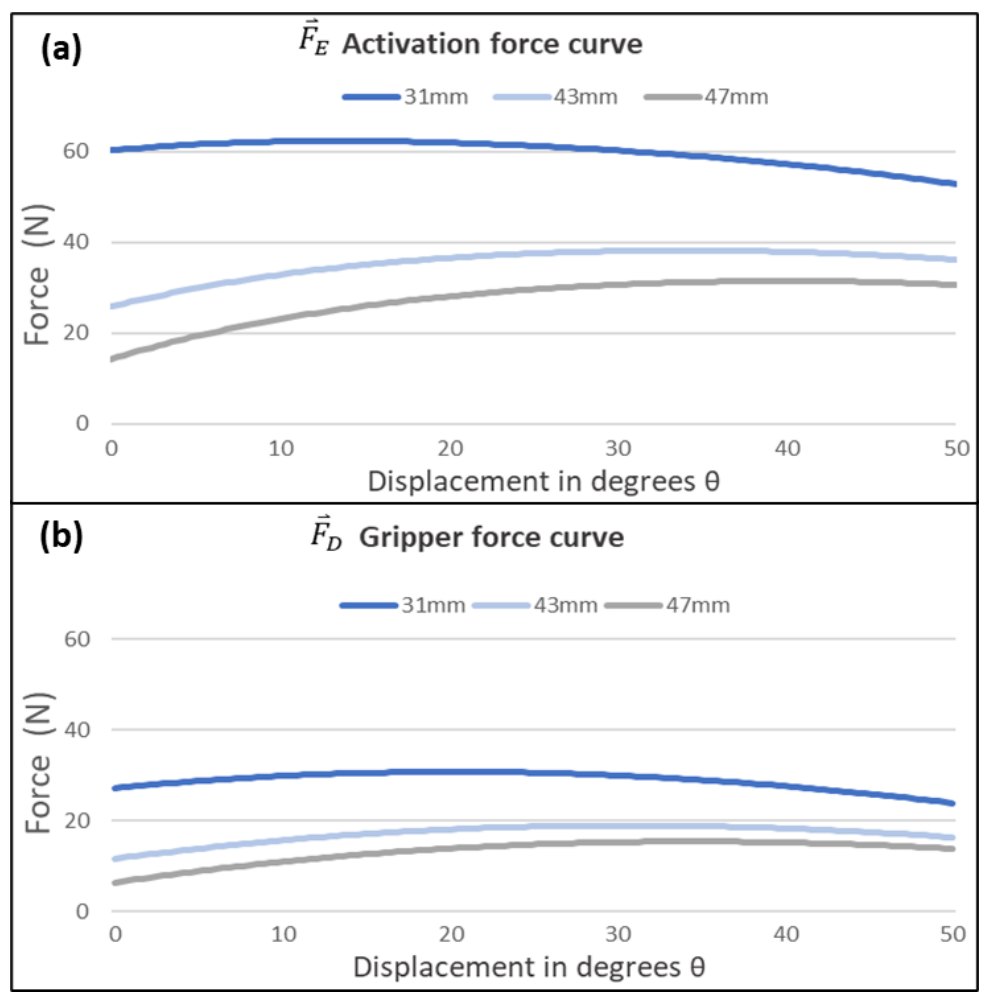

Figure 5. (a) Activation force curve and (b) gripper force curve for unstretched band lengths of $31 \mathrm{~mm}, 43 \mathrm{~mm}$, and $47 \mathrm{~mm}$. Note: curves do not represent continuous measurement but discrete interpolated samples.

\subsubsection{Design for Manufacturing}

The in-house manufactured parts were slightly modified to achieve draft angles that would facilitate the manufacturing process. In areas where it was not possible to modify the negative draft angle, molds were devised to allow a smoother removal of the part. The tolerances were defined using GD\&T as a result of stack up tolerances for the assembled parts. The carbon fiber parts presented in Figure 6 were created from soft molds, which, in turn, were created from hard molds by a Roland model MDX-40A CNC.

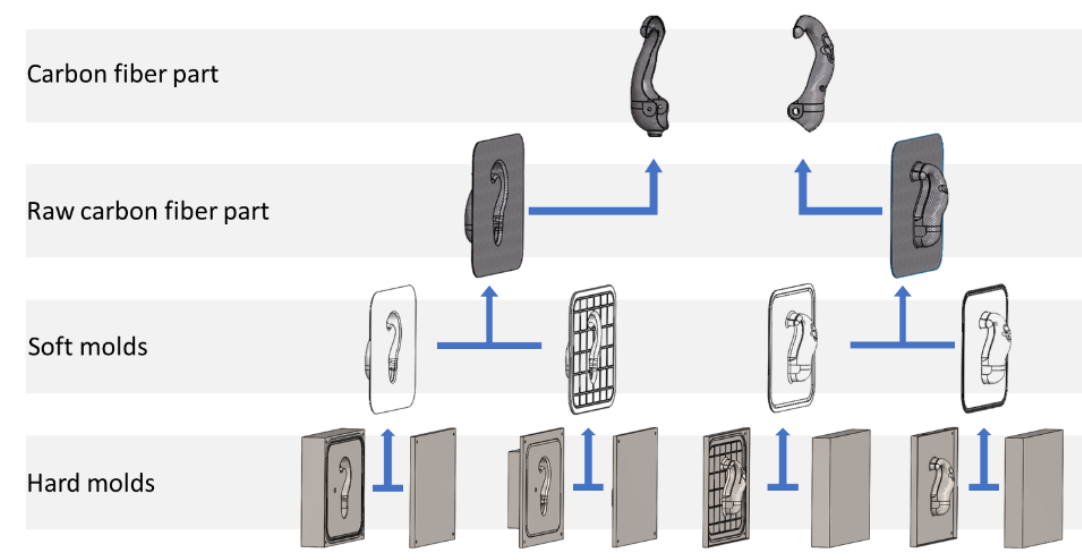

Figure 6. Design of molds to generate carbon fiber parts.

\subsubsection{Manufacturing}

The in-house manufactured parts were performed at lab-scale using System 2000 Fibreglast epoxy resin and carbon fiber $3 \mathrm{~K} 2 \times 2$ twill weave. Figure 7 a shows a raw carbon fiber part made from a molding process performed in an autoclave, which was placed in fixture for its final cutout (Figure 7b). The silicone parts presented in Figure $7 \mathrm{c}$ were 
manufactured using acrylic molds by a molding cast performed in an autoclave. Finally, Figure $7 \mathrm{~d}$ shows the final prototype, which was assembled using in-house manufacturing and purchased parts.
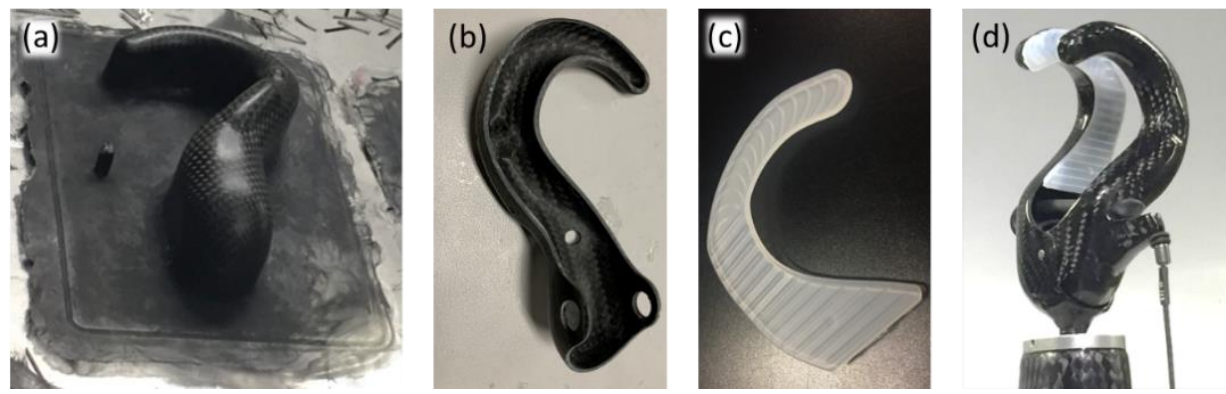

Figure 7. (a) Raw carbon fiber part, (b) carbon fiber trimmed, (c) silicone rubber, and (d) final prototype.

\subsection{Requirements Evaluation}

\subsubsection{Mass}

Weight reduction is a high priority for individuals of all ages and wearers of all types of prostheses [1]; therefore, our research was focused on weight reduction through the use of ultralight materials with high mechanical resistance, combined with a structurally adequate design. As a result, a body-powered hook (Carbon HK VO hook) with a mass of $100 \mathrm{~g}$ was achieved, which is even lighter than current commercially available alternatives (Figure 8); the Hosmer 5X weighs $213 \mathrm{~g}$ [7] and high-tech myoelectric prostheses can exceed $600 \mathrm{~g}$ [4]. Clearly, a body-powered hook prosthesis-due to its simplicity-is lighter than a myoelectric prosthesis; therefore, its comparison seems unfair. However, it is not, since all prostheses, regardless of their technology, are worn by a user, and their weight is supported by that user's stump. In this manner, the development of a lightweight and comfortable prosthesis is the most important design priority [1]. The Carbon HK VO hook weighs less than the $20 \%$ of the total weight of an electric prosthesis, making it more comfortable and functional for a longer time.

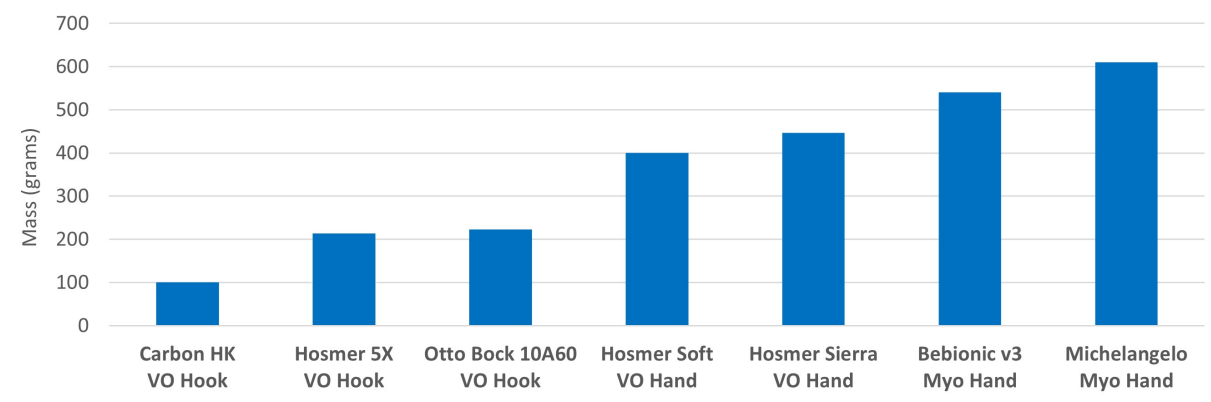

Figure 8. Carbon HK VO hook mass (left) compared to current state-of-the-art commercially available upper limb prosthetics.

\subsubsection{Functional Design}

The Carbon HK VO hook developed in this study has a simple design. The hook performs basic grip and tip pinch grasp functions, which allow users to perform the highest number of daily tasks possible. The clamp has a maximum opening capacity of $84 \mathrm{~mm}$, which allows the user to hold various objects, from small coins to large cups of coffee (Figure 9a). Its hook shape allows users to hold bags without activating the prosthesis; moreover, it is possible to push objects or press small buttons with the tip. The Carbon HK VO hook can also firmly hold objects with a handle using a three-point clamp, such as pens or hand tools. Finally, the user can regulate the force level as desired (Figure 9b), allowing them to hold delicate objects without breaking them or heavy objects without losing grip. 


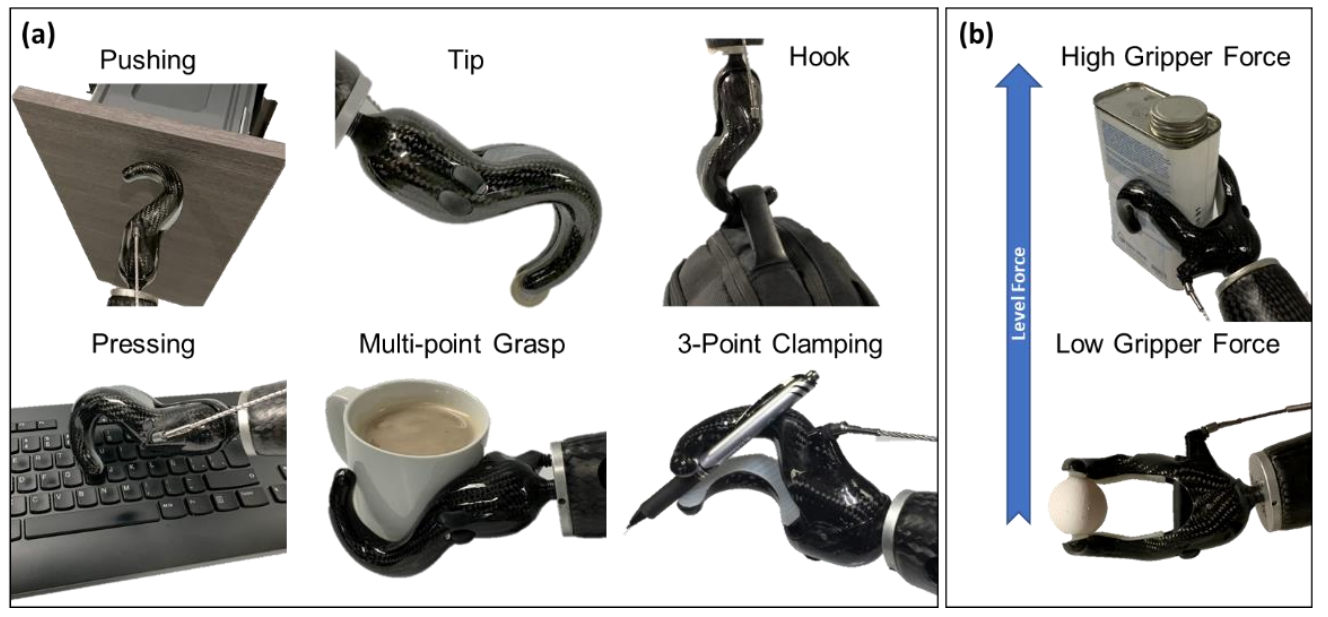

Figure 9. Functional design: (a) functions and (b) level force.

\subsubsection{Grip Force Regulation System}

Prostheses with a grip force above $20 \mathrm{~N}$ enable basic daily-living activities [14]. However, not all of these activities require the same grip force; therefore, the regulation of grip force is an important factor to be considered for achieving a functional device. The typical grip force regulation systems presented in commercially available alternatives are limited. For example, grip force regulation in the Hosmer 5XA VO hook is achieved by placing additional bands [14]. This system increases the number of components, resulting in a complicated system for users that only achieves discrete regulation. Similarly, force regulation on the Ottobock 10A60 hook is achieved by a lever that varies the kinematics of the mechanism in two different positions, allowing only two levels of force [14].

In contrast, the Carbon HK VO hook developed in this study was designed with a continuous force regulation system. Force is generated by a typical tubular commercial band held by friction from point $\mathrm{C}$ to point $\mathrm{H}$ (Figure 4), which has an initial distance of $52 \mathrm{~mm}$ at $0^{\circ} \theta$. As shown in Figure 10, tension variation is achieved when the user tenses or releases the tubular band. The continuous regulation system, as one of the main characteristics of the Carbon HK VO hook, increases device functionality and provides advantages over similar devices. In addition, the great diversity of available tubular bands on the market with different levels of resistance allows the user to select one at their convenience and easily replace it when it degrades.

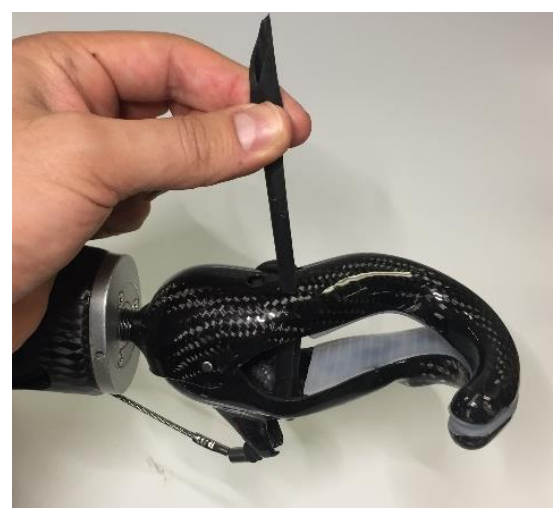

Figure 10. Continuous force regulation system.

\subsubsection{Activation Force}

To avoid fatigue, maximum activation force levels of $38 \mathrm{~N}$ and $66 \mathrm{~N}$, for the average female and the average male, respectively, were established [21]. Figure 5a shows that, when a male user applies an unstretched length of $31 \mathrm{~mm}$, the activation force is lower than 
$66 \mathrm{~N}$, with a maximum grip force of 30 N. Similarly, a female user applying an unstretched length of $43 \mathrm{~mm}$ presents an activation force of $38 \mathrm{~N}$, obtaining a maximum grip force of $18 \mathrm{~N}$. Whether or not female and male users desire to use the device below the fatigue line, an unstretched length of $47 \mathrm{~mm}$ can be used to obtain a maximum grip force of $15 \mathrm{~N}$.

\subsubsection{Materials}

Carbon fiber was selected as the structural material in order to reduce weight while maintaining high mechanical resistance. The composite material presented in Figure 11a was constituted by System 2000 Fibreglast epoxy resin and carbon fiber $3 \mathrm{~K}, 2 \times 2$ twill weave. All carbon fiber was covered with the polyester resin Duratec, which provides UV protection and high-gloss cosmetics properties. This commercial resin was enriched with titanium dioxide nanoparticles $\left(\mathrm{TiO}_{2}\right)$ in order to achieve hydrophobic properties. The contact angle was measured with OCA 15 PLUS Dataphysics equipment. The rectangular substrates of the treated carbon fiber, with a typical size of $1 \times 0.5^{\prime \prime}$, were homogeneously covered with water droplets, which were stable and kept their spherical shape (Figure $11 \mathrm{~b}, \mathrm{c}$ ). According to the measurements, the average contact angle was equal to $116^{\circ} \pm 2^{\circ}$ and the maximum contact angle was $128^{\circ} \pm 3^{\circ}$, matching the water repellence standard established for the material studied [33]. The average surface tension was equal to $72.7 \times 0.1 \mathrm{mN} / \mathrm{m}$. Figure $11 \mathrm{~d}$,e shows AFM images of the substrate presenting a marked relief on a sub-micron scale, with an RMS roughness of $299.8 \mathrm{~nm}$ and an average roughness of $245.2 \mathrm{~nm}$. These surface characteristics agree with the obtained hydrophobic properties of the material.

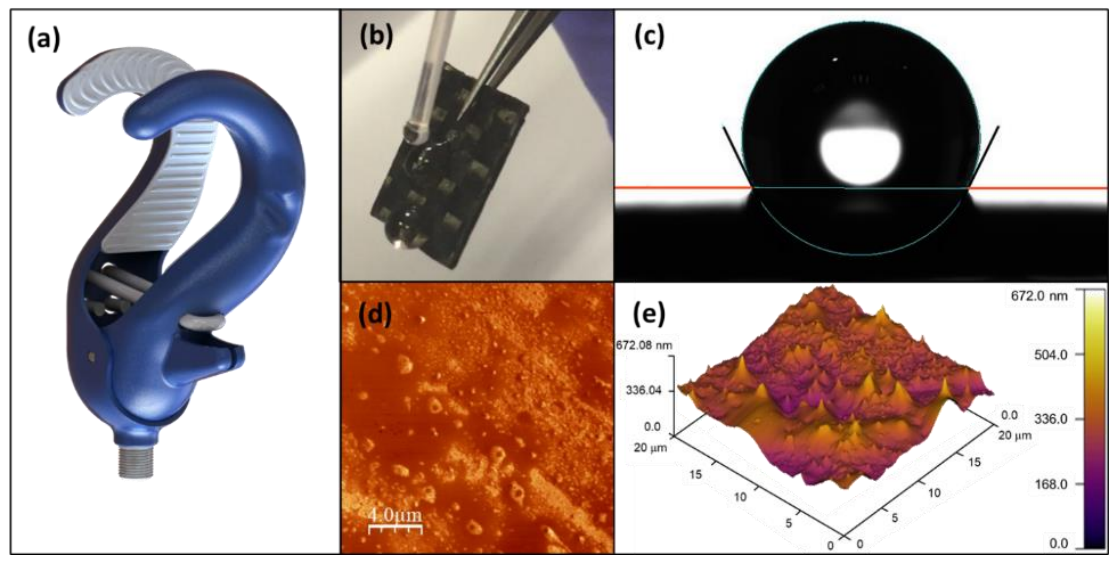

Figure 11. Surface properties of chemically treated carbon fiber: (a) carbon fiber material (blue) covered with hydrophobic polyester resin; (b) water repellence observed by depositing droplets on the surface; (c) characteristic image of a droplet with contact angles marked; (d,e) AFM images (2D and $3 \mathrm{D}$ representations, respectively) of the treated carbon fiber, showing details of surface relief and roughness.

Silicone is a widely used material for the handling of food products because it is totally inert and resists extreme temperatures. In addition, its non-slip properties caused by a high coefficient of friction provides an excellent grip. Therefore, silicone was selected as the material for the inner surfaces (Figure 12a), since this area of the Carbon HK VO hook is in contact with different objects, including food products. At this stage, a lab-scale prototype, Dragon Skin 30 silicone rubber, was used. This commercial silicone was enriched with silver nanoparticles (nSi@AgNPs) in order to achieve antibacterial properties. Figure 12b-e shows the pathway followed in the design of the antibacterial silicon grip. The process begins with the synthesis of nSi@AgNPs (powder), which is then incorporated into the silicone matrix (uncured silicone) to obtain a cured antibacterial silicone in the labsite and the final antibacterial silicone grip prototype. The AFM images presented in Figure 12f,g show a homogeneous dispersion of nSi@AgNPs on the silicone surface, as well as a mean roughness in the nanometric scale. In this way, our antibacterial silicone helps to keep the 
silicone grip free of bacteria, according to the antibacterial activity tested against $E$. coli and S. aureus as Gram-negative and Gram-positive bacteria models, respectively.

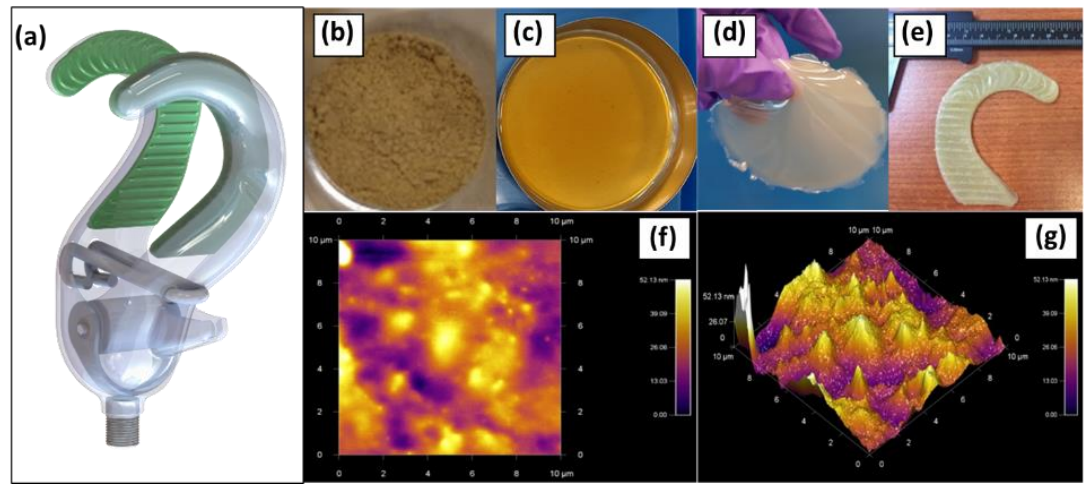

Figure 12. (a) Silicone rubber material with antibacterial properties (green). Pathway for obtaining the antibacterial silicone grip: (b) synthesis of nSi@AgNPs powder; (c) incorporation of nSi@AgNPs into the silicone matrix; (d) cured antibacterial silicone in the labsite; (e) antibacterial silicone grip prototype; (f) 2D, and (g) 3D AFM images of the surface of the antibacterial silicone grip prototype.

\subsubsection{Robustness}

The materials used for the manufacturing of this body-powered hook are carbon fiber, silicone, stainless steel, ABS (acrylonitrile butadiene styrene), and bronze; these materials provide resistance to dust, water, and temperatures around $5{ }^{\circ} \mathrm{C}$ to $70{ }^{\circ} \mathrm{C}$ (limited by the rubber band). The parts and materials are identified in Figure 13. In contrast to myoelectrical prostheses, which cannot be immersed in liquids, the Carbon HK VO hook is resistant to liquids since it has no electronic components or small mechanical parts. The developed device weighs less than half of the Hosmer $5 X$, which is made of stainless steel. Yet, the Carbon HK VO hook has a similar weight to the Hosmer 5XA, which is made of aluminum; however, according to the product information, this commercial hook is used for light to moderate duty [34]. The Carbon HK VO hook achieves greater robustness than both Hosmer models, since it is made of carbon fiber with $E \approx 150-500 \mathrm{GPa}$, in contrast to aluminum with $E \approx 70 \mathrm{GPa}$ and steel with $E \approx 200 \mathrm{GPa}$. In this way, greater levels of stiffness can be achieved with less steel or aluminum weight.

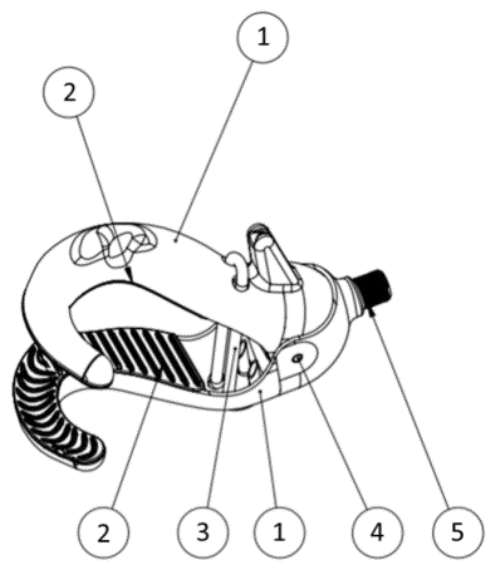

Figure 13. Device parts: (1) two carbon fiber pliers; (2) two non-slip silicone rubbers; (3) latex tubular band; (4) stainless steel shaft with ABS plastic coating; and (5) stainless steel fine thread.

\subsubsection{Ease of Installation}

Ease of installation is related to the prosthetist, who typically installs the prosthesis through a socket, specifically made for the stump of the user. A stump socket for myoelectric prosthesis requires a complex manufacture, since it must contain myoelectric sensors 
and batteries; in contrast, a stump socket for a body-powered prosthesis is simpler, as it does not require additional elements. A screw of $0.5^{\prime \prime}$, which is widely used in commercial body-powered prostheses, was used in this device in order to connect the prosthesis to the socket of the stump. In this way, the connection is economical, robust, and easy for prosthetists. This feature allows this new device to be placed in existent stump sockets, as it does not require a special stump socket.

\subsubsection{Appearance}

The device has an organic design free of sharp edges made of carbon fiber, which is a material widely used in luxury products for its nice appearance. To improve its appearance, the carbon fiber was covered with a high gloss resin. The insulating properties of the carbon fiber provide more comfort to the user, since it is not cold to the touch.

\subsubsection{Affordability}

Although $80 \%$ of the more than 600 million people with disabilities live in developing countries [2,3], the development of medical devices usually only considers the needs and infrastructure of people from high-income countries [2]. As an example, prostheses such as Michelangelo, BeBionic and i-Limb, which all cost over USD 50,000, are not feasible for a typical user from a low-income country, not only because of their high cost but also because it is not robust enough for their day-to-day functions and because its installation requires specialized personnel. In a low-income scenario, active prosthetic options are limited to body-powered technologies. The Hosmer 5X hook, which is used widely by people in low-income countries, has an approximate value of USD 500, which is affordable for a public health system in a developing country.

The manufacturing cost of $1 \mathrm{~kg}$ of carbon fiber for large-scale production $(>50,000$ units) in 2020 is estimated at USD $10 / \mathrm{kg}$. The cost of the material is $55 \%$ of the total cost, while the remaining $45 \%$ corresponds to labor, tools, equipment, and overhead [35]. Similarly, for low-scale production (<200 units), the manufacturing cost increases to USD $50 / \mathrm{kg}$. Considering these costs and the weight of the Carbon HK VO hook (100 g) presented in this study, it has an estimated manufacturing cost of USD 1-5 for the total amount of carbon fiber parts in the device, corresponding to $80 \%$ of the product weight. Although this cost does not include nanoparticles coating, profit, transportation, administrative costs, or taxes, this prosthesis is estimated to have a commercial cost of USD $\leq \$ 500$ per unit, an affordable cost for developing countries.

\subsection{Comparison with Commercial Prostheses and Research Developments}

Table 2 presents a summary of the specifications of the developed Carbon HK VO hook in comparison with the Hosmer 5X hook, which is the most commonly used prosthesis in developing countries, and the Ottobock Michelangelo, as an example of a high-tech myoelectric prosthesis. It can be seen that the carbon fiber used as the main material reduces the weight of the device considerably in comparison to the Ottobock, while the continuous regulation system allows for a higher functionality compared to the Hosmer $5 \mathrm{X}$ hook.

Table 2. Specifications of the Carbon HK VO Hook compared to current available upper limbs prosthetic.

\begin{tabular}{|c|c|c|c|}
\hline Requirement & $\begin{array}{c}\text { (This Study) Carbon HK VO } \\
\text { Hook }\end{array}$ & Hosmer Model 5X Hook & Ottobock Michelangelo \\
\hline Mass & $100 \mathrm{~g}$ & $213 \mathrm{~g}[13]$ & $>600 \mathrm{~g}[9]$ \\
\hline Degree of freedom & 1 & 1 & 2 \\
\hline Gripping force regulation & Continuous regulation system & Discrete regulation system & Continuous regulation system \\
\hline Activation force & Body-powered & Body-powered & Electric/Myoelectric \\
\hline Main material & Carbon fiber & Stainless steel & NA \\
\hline Robustness (works submerged in water) & Yes & Yes & No \\
\hline Ease of installation & Easy & Easy & Hard \\
\hline Affordability (Commercial price) & $<500$ USD & $<500$ USD & $>50000$ USD \\
\hline
\end{tabular}


Force regulation is of great relevance for a prosthesis, since not all objects must be held with the same force. Similar developments, such as the Hosmer 5X, presents the possibility of adding bands to vary force [14]; however, that results in a complex maneuver and additional efforts when constantly adding bands. In the same regard, the Ottobock Model 10A60 Hook only has two levels of force [14]. Yet, users have a total control of the grip force on developments with VC mechanism, such as "GRIP 5"; however, discomfort is frequently reported. Contrarily, the continuous force regulation system presented in this study proposes the use of a simple mechanism with no additional components that could increase the total prosthesis's weight.

In order to develop a feasible prosthesis for users, its manufacturing process must be considered. In this regard, a lot of research has been focused on 3D-printed prostheses, presenting them as "affordable alternatives"; however, the additive manufacturing is hard to scale for a larger production. In addition, most of these prostheses are manufactured by FDM (fused deposition modeling) technology, which generates pieces with porous surfaces. The presence of pores favors the accumulation of dirt, viruses, and bacteria, which is not favorable for a hand prosthesis, since cleanliness is of great importance. In contrast, the Carbon HK VO hook presented in this study allows for a manufacturing process that is easily scalable with hydrophobic and antibacterial properties.

As in our study, the "Delft Cylinder Hand" [23] was also developed considering the design requirements of users, achieving a mass reduction with a simple design manufactured in aluminum. Despite having different terminal devices, both body-powered developments ("Delft Cylinder Hand" and "Carbon $\mathrm{HK}^{\prime}$ ) achieved significant improvements concerning comfortability by following different strategies.

In summary, a lightweight, robust, and functional device at an affordable cost has been developed as an appropriate medical device considering the needs and context of the majority of users.

\subsection{Study Limitations}

In this study, five Carbon HK VO hook devices were manufactured, which were only tested within the laboratory. The Carbon HK VO hook presented in this study has a simple design intended to perform the basic hook grip and tip pinch grasp functions; therefore, complex functions are not feasible. Yet hydrophobic properties are acquired by the coating of nanoparticles on the outer layer, which could be reduced by daily use. Continuous adjustments to band tension could degrade it prematurely, since the regulation system uses friction to hold the band. However, there are many tubular bands on the market, and it can be easily replaced. In addition, no user was asked to test the devices. For further research, an extensive clinical trial is required to evaluate the performance of the Carbon $\mathrm{HK} \mathrm{VO}$ hook in everyday situations outside the laboratory. An evaluation with users is planned to be undertaken in collaboration with Mexican public health institutions.

\section{Conclusions}

The upper-limb prosthesis developed in this study was intended to be an appropriate alternative for developing and developed countries' populations. Through the I-corps program, the body-powered hook was selected to be developed due to its high functionality, low weight, low cost, and low maintenance compared to myoelectric and body-powered hand prostheses. Then, a list of design requirements was established through a literature review. This work shows the development of the device from its conceptual design, detailed design, design for manufacturing, and, finally, the manufacture of the prototype with the characteristics necessary for large-scale manufacturing.

As a result, the Carbon HK VO hook was carefully designed to achieve the most important user design criteria, including light weight and functionality. The Carbon HK VO hook weighs $100 \mathrm{~g}$, making it one of the lightest prostheses available. Carbon fiber was selected as the structural material, which is widely used in medical devices because of its high resistance, its light weight, and its attractive appearance. In order to increase user 
comfort, this device presents a novel continuous force regulation system, which allows a griping force from 0 to $30 \mathrm{~N}$. In addition, its body-powered actuation provides the user with proprioceptive force, which enables accurate and fast control. Regarding the sanitization of the device, the device was made with materials with antibacterial and hydrophobic properties.

\section{Patents}

Patent pending, application number: MX/u/2020/000264.

Author Contributions: Conceptualization, M.A.T.-L.; Formal Analysis, D.A.R.-S.; Funding Acquisition, M.A.T.-L.; Investigation, M.A.T.-L., A.M.A.-M., T.E.L.-C., and J.B.-C.; Methodology, M.A.T.-L.; Project Administration, M.A.T.-L.; Writing_-Original Draft, R.B.G.-G.; Writing—Review \& Editing, A.I.P.-S. and J.A.C.-R. All authors have read and agreed to the published version of the manuscript.

Funding: This research was funded by FONCICYT, grant number C0014-2016-04.

Institutional Review Board Statement: Not applicable.

Informed Consent Statement: Not applicable.

Data Availability Statement: Not applicable.

Acknowledgments: The support of medical appliance technicians are acknowledged: Joaquin Oropeza, for his great advice on the design of this device, as well as Marlo Ortiz and Carlos Ramos Vizcaino for their support in the construction of the stump socket for first-time trial users. The collaboration of undergraduate students is also acknowledged: Carolina Contreras, Andrea Rodríguez, Andrea Verástegui, Andrea Flores, and Mara Padilla. Finally, the participation of Emilio, Brenda, and Jorge as users is also acknowledged.

Conflicts of Interest: The authors declare no conflict of interest.

\begin{tabular}{ll}
\multicolumn{2}{l}{ Abbreviations } \\
2D & Two-dimensional \\
3D & Three-dimensional \\
4 A's & Availability, accessibility, appropriateness, and affordability \\
ABS & Acrylonitrile butadiene styrene \\
AFM & Atomic force microscopy \\
AISI & American Iron and Steel Institute \\
Carbon HK & Body-powered hook developed in this study \\
FDM & Fused deposition modeling \\
GD\&T & Geometric dimensioning and tolerancing \\
I-Corps & National Science Foundation's Innovation Corps \\
IMSS & Mexican Institute of Social Security \\
INR & National Rehabilitation Institute \\
NSF & National Science Foundation \\
nSi@AgNPs & Commercial silicone enriched with silver nanoparticles \\
RMS & Root Mean Square \\
TiO2 & Titanium dioxide nanoparticles \\
USD & United states dollar \\
UV & Ultraviolet \\
VC & Voluntarily closed \\
VO & Voluntarily open \\
&
\end{tabular}

\section{References}

1. Biddiss, E.; Beaton, D.; Chau, T. Consumer design priorities for upper limb prosthetics. Disabil. Rehabil. Assist. Technol. 2007, 2, 346-357. [CrossRef]

2. Organización Mundial de la Salud Dispositivos médicos: La gestión de la discordancia. World Heal. Organ. 2012, 1, 128.

3. World Health Organization (WHO) WHO global disability action plan 2014-2021: Better health for all people. World Heal. Organ. 2015, 1-25. 
4. Belter, J.T.; Segil, J.L.; Dollar, A.M.; Weir, R.F. Mechanical design and performance specifications of anthropomorphic prosthetic hands: A review. J. Rehabil. Res. Dev. 2013, 50, 599-618. [CrossRef]

5. World Health Organization. Medical Devices: Managing the Mismatch: An Outcome of the Priority Medical Devices Project; World Health Organization: Geneva, Switzerland, 2010; pp. 1-36.

6. Biddiss, E.; Chau, T. Upper limb prosthesis use and abandonment: A survey of the last 25 years. Prosthet. Orthot. Int. 2007, 31, 236-257. [CrossRef]

7. Belter, J.T.; Reynolds, B.C.; Dollar, A.M. Grasp and force based taxonomy of split-hook prosthetic terminal devices. $201436 t h$ Annu. Int. Conf. IEEE Eng. Med. Biol. Soc. EMBC 2014 2014, 6613-6618. [CrossRef]

8. Kejlaa, G.H. Consumer concerns and the functional value of prostheses to upper limb amputees. Prosthet. Orthot. Int. 1993, 17, 157-163. [CrossRef]

9. Riener, R. The Cybathlon promotes the development of assistive technology for people with physical disabilities. J. Neuroeng. Rehabil. 2016, 13, 2-5. [CrossRef]

10. Ottobock worldwide - Innovations that Move People I Ottobock SE \& Co. KGaA. Available online: https://www.ottobock.com/ en/ (accessed on 2 April 2021).

11. Össur. Life Without Limitations. Available online: https:/ / www.ossur.com/region-selector (accessed on 2 April 2021).

12. Powered Arm Prosthesis Race ICYBATHLON ETH Zürich. Available online: https://cybathlon.ethz.ch/en/event/disciplines/ arm (accessed on 2 April 2021).

13. Smit, G.; Plettenburg, D.H. Efficiency of voluntary closing hand and hook prostheses. Prosthet. Orthot. Int. 2010, 34, 411-427. [CrossRef] [PubMed]

14. Smit, G.; Bongers, R.M.; Van der Sluis, C.K.; Plettenburg, D.H. Efficiency of voluntary opening hand and hook prosthetic devices: 24 years of development? J. Rehabil. Res. Dev. 2012, 49, 523-534. [CrossRef] [PubMed]

15. Millstein, S.G.; Heger, H.; Hunter, G.A. Prosthetic use in adult upper limb amputees: A comparison of the body powered and electrically powered prostheses. Prosthet. Orthot. Int. 1986, 10, 27-34. [CrossRef]

16. ten Kate, J.; Smit, G.; Breedveld, P. 3D-printed upper limb prostheses: A review. Disabil. Rehabil. Assist. Technol. 2017, 12, 300-314. [CrossRef] [PubMed]

17. Frey, D.D.; Carlson, L.E.; Ramaswamy, V. Voluntary-opening prehensors with adjustable grip force. J. Prosthet. Orthot. 1995, 7 124-131. Available online: Voluntary_Opening_Prehensors_with_Adjustable_Grip.4.pdf (accessed on 2 April 2021). [CrossRef]

18. Berning, K.; Cohick, S.; Johnson, R.; Miller, L.A.; Sensinger, J.W. Comparison of body-powered voluntary opening and voluntary closing prehensor for activities of daily life. J. Rehabil. Res. Dev. 2014, 51, 253-262. [CrossRef] [PubMed]

19. FISHMAN, S.; BERGER, N. The choice of terminal devices. Artif. Limbs 1955, 2, 66-77. [PubMed]

20. Atkins, D.J.; Heard, D.C.Y.; Donovan, W.H. Epidemiologic Overview of Individuals with Upper-Limb Loss and Their Reported Research Priorities. J. Proshetics Orthot. 1996, 8, 2-11. [CrossRef]

21. Hichert, M.; Vardy, A.N.; Plettenburg, D. Fatigue-free operation of most body-powered prostheses not feasible for majority of users with trans-radial deficiency. Prosthet. Orthot. Int. 2018, 42, 84-92. [CrossRef]

22. Home I Maker Hand. Available online: https:/ / www.makerhand.com/ (accessed on 21 April 2021).

23. Smit, G.; Plettenburg, D.H.; Van Der Helm, F.C.T. The Lightweight Delft Cylinder Hand, the First Multi-Articulating Hand That Meets the Basic User Requirements. IEEE Trans. Neural Syst. Rehabil. Eng. 2014, 23, 431-440. [CrossRef]

24. Grip 5 Evolution Prehensor I Prosthetic Hand I International Shipping. Available online: https://www.trsprosthetics.com/ product/grip-5-evolution-prehensor/ (accessed on 21 April 2021).

25. Bahari, M.S.; Jaffar, A.; Low, C.Y.; Jaafar, R.; Roese, K.; Yussof, H. Design and Development of a Multifingered Prosthetic Hand. Int. J. Soc. Robot. 2012, 4, 59-66. [CrossRef]

26. Gretsch, K.F.; Lather, H.D.; Peddada, K.V.; Deeken, C.R.; Wall, L.B.; Goldfarb, C.A. Development of novel 3D-printed robotic prosthetic for transradial amputees. Prosthet. Orthot. Int. 2016, 40, 400-403. [CrossRef]

27. Simone, F.; York, A.; Seelecke, S. Design and fabrication of a three-finger prosthetic hand using SMA muscle wires. In Bioinspiration, Biomimetics, and Bioreplication 2015; International Society for Optics and Photonics: Bellingham, WA, USA, 2015. [CrossRef]

28. Doubrovski, Z.; Verlinden, J.C.; Geraedts, J.M.P. Optimal Design for Additive Manufacturing: Opportunities and Challenges. DETC2011-4 2016, 1-12. [CrossRef]

29. Walker, M.J.; Goddard, E.; Stephens-Fripp, B.; Alici, G. Towards Including End-Users in the Design of Prosthetic Hands: Ethical Analysis of a Survey of Australians with Upper-Limb Difference. Sci. Eng. Ethics 2020, 26, 981-1007. [CrossRef] [PubMed]

30. Reynolds, H.M. Investigation of Inertial Properties of the Human Body W. \& OfPARIMMT OF \% Owe " labial Techmcal luwuu S-rnce. 2015. Available online: https:/ /apps.dtic.mil/dtic/tr/fulltext/u2/a016485.pdf (accessed on 6 May 2021).

31. Product Archive I Fillauer LLC I Orthotics and Prosthetics Manufacturer. Available online: https://fillauer.com/products/ (accessed on 2 April 2021).

32. Jones, L. Dextrous Hands: Human, Prosthetic, and Robotic. Teleoper. Virtual Environ. 1997, 6, 29-56. [CrossRef] [PubMed]

33. Arnold, C.L.; Eyckens, D.J.; Servinis, L.; Nave, M.D.; Yin, H.; Marceau, R.K.W.; Pinson, J.; Demir, B.; Walsh, T.R.; Henderson, L.C. Simultaneously increasing the hydrophobicity and interfacial adhesion of carbon fibres: A simple pathway to install passive functionality into composites. J. Mater. Chem. A 2019, 7, 13483-13494. [CrossRef] 
34. Hosmer Prosthetic Model 5XA Hook: Aluminum, Nitrile Rubber I Amputee Store. Available online: https://amputeestore.com/ products/hosmer-model-5xa-prosthetic-hook (accessed on 3 April 2021).

35. Shama Rao, N.; Simha, T.G.A.; Rao, K.P.; Ravi Kumar, G.V.V. Carbon Composites are Becoming Competitive and Cost Effective; Infosys Ltd. 2018, p. 1. Available online: https:/ / www.infosys.com/engineering-services/white-papers/documents/carboncomposites-cost-effective.pdf (accessed on 22 June 2018). 